\title{
Artificial Intelligence Based Clustering with Routing Protocol for Internet of Vehicles
}

\section{Manar Ahmed Hamza ${ }^{1, *}$, Haya Mesfer Alshahrani ${ }^{2}$, Fahd N. Al-Wesabi ${ }^{3,4}$, Mesfer Al Duhayyim ${ }^{5}$, Anwer Mustafa Hilal ${ }^{1}$ and Hany Mahgoub ${ }^{3}$}

\author{
${ }^{1}$ Department of Computer and Self Development, Preparatory Year Deanship, Prince Sattam bin Abdulaziz University, \\ Alkharj, Saudi Arabia \\ ${ }^{2}$ Department of Information Systems, College of Computer and Information Sciences, Princess Nourah Bint Abdulrahman \\ University, Saudi Arabia \\ ${ }^{3}$ Department of Computer Science, King Khalid University, Muhayel Aseer, Saudi Arabia \\ ${ }^{4}$ Faculty of Computer and IT, Sana'a University, Sana'a, Yemen \\ ${ }^{5}$ Department of Natural and Applied Sciences, College of Community-Aflaj, Prince Sattam bin Abdulaziz University, \\ Saudi Arabia \\ *Corresponding Author: Manar Ahmed Hamza. Email: ma.hamza@psau.edu.sa \\ Received: 21 June 2021; Accepted: 28 July 2021
}

\begin{abstract}
With recent advances made in Internet of Vehicles (IoV) and Cloud Computing (CC), the Intelligent Transportation Systems (ITS) find it advantageous in terms of improvement in quality and interactivity of urban transportation service, mitigation of costs incurred, reduction in resource utilization, and improvement in traffic management capabilities. Many trafficrelated problems in future smart cities can be sorted out with the incorporation of IoV in transportation. IoV communication enables the collection and distribution of real-time essential data regarding road network condition. In this scenario, energy-efficient and reliable intercommunication routes are essential among vehicular nodes in sustainable urban computing. With this motivation, the current research article presents a new Artificial Intelligence-based Energy Efficient Clustering with Routing (AI-EECR) Protocol for IoV in urban computing. The proposed AI-EECR protocol operates under three stages namely, network initialization, Cluster Head $(\mathrm{CH})$ selection, and routing protocol. The presented AI-EECR protocol determines the CHs from vehicles with the help of Quantum Chemical Reaction Optimization (QCRO) algorithm. QCRO algorithm derives a fitness function with the help of vehicle speed, trust level, and energy level of the vehicle. In order to make appropriate routing decisions, a set of relay nodes was selected using Group Teaching Optimization Algorithm (GTOA). The performance of the presented AI-EECR model, in terms of energy efficiency, was validated against different aspects and a brief comparative analysis was conducted. The experimental outcomes established
\end{abstract}


that AI-EECR model outperformed the existing methods under different measures.

Keywords: Vehicular communication; internet of vehicles; energy efficient; smart transportation; smart city; urban computing

\section{Introduction}

Transportation is one of the basic activities for effective functioning of cities. The concept of cities has been greatly influenced by prosperity and development of transportation. Transportation and urban system developments are significantly correlated to each other. The concepts such as location of the city and land rent demonstrates the relationship between urban land and transport utilization [1,2]. In order to provide sufficient transportation facilities, with restricted assets in cities, Intelligent Transportation System (ITS) has been developed. ITS employs Information and Communication Technology (ICT) to effectively use the existing transport framework with goals such as enhancing transportation facilities and decrease air pollution, congestion, and accidents. In the past two decades, the idea of "smart cities" have attained more popularity, thanks to phenomenal growth of ICT and intense support from huge technology vendors. Several cities across the globe started carrying out general methods to improve urban facilities, utilizing techniques in terms of smart city enterprises.

In spite of distinct emphases made upon and descriptions about the label 'smart city' [3], the application of techniques in enhancing the standards of living forms the crux. Energy efficient solutions and reduction of emissions have become significant factors for smart transportation in urban computing. Indeed, energy saving is considered as the main advantage and is generally estimated through cost value analyses so as to justify the investment made in ITS. In fact, transportation accounts for $1 / 5^{\text {th }}$ of energy utilization across the globe, with customer road transport being the large allocation. But it is complex to effectively decrease the usage of energy spent on transportation in general and urban regions. Urban transportation requires management which is enabled by ITS so as to achieve the main result i.e., reduction of climate changes [4]. In a sustainable environment i.e., reduction of climate changes and low energy consumption, ITS investment becomes a major part in the context of a smart city. So, it is critical to understand the technological and institutional situations of energy saving advantage.

Internet of Vehicles (IoV) is an innovative form of classical Vehicular Ad-Hoc Network (VANET) based on applicability, scalability, and infrastructure. This progression leads to the improvement of several communications that occur at road level between infrastructure and humans [5,6]. Numerous investigations have developed several applications and methods like traffic management, road safety, network congestion, routing strategies, and data distribution [7]. In relation to VANET, IoV has an advantage i.e., extending the application of ITS in several research fields and industries [8,9]. Further, it is easy to implement and has quick access to internet as well. IoV allows the allocation of data amongst vehicles and provides useful information such as parking details, fuel stations, availability of hotels, and other information. Another advantage is its ability to preserve information on several related vehicles (i.e., scalability).

\subsection{Energy Efficient Solutions for Smart Transportation}

A number of research works has been conducted upon vehicular packet transmission and clustering to enhance the energy efficiency of network [10,11]. In fact, clustering simplifies the stable and scalable network frameworks and transmission. It has a goal i.e., to group the vehicles 
under different number of clusters based on specific procedures. Every vehicle in cluster framework could perform distinct operations like Cluster Head $(\mathrm{CH})$ and Cluster Member $(\mathrm{CM}) /$ gateway. In clustering, $\mathrm{CH}$ performs as an accessing point and manages traffic control and the performance of QoS. Each vehicle interacts straightaway in one cluster $[12,13]$ or else, the interaction that occurs among the vehicles should depend on CHs. The volume of data utilized to store the network conditions gets decreased when using clustering method. Similarly, routing depends upon clustering as it enhances the ability of IoV. In fact, clustering generates a virtual backbone for transmission for efficient supply of information in IoV and it also exploits the rare assets, for instance, bandwidth. Assuming that the vehicles on road could be made as cluster, routing depends upon clustering, an appropriate one for VANET.

\subsection{Paper Contributions}

The current research article presents a new Artificial Intelligence based Energy Efficient Clustering with Routing (AI-EECR) protocol for IoV in urban computing. The proposed AI-EECR protocol operates under three stages namely, network initialization, Cluster Head $(\mathrm{CH})$ selection, cluster construction, and routing protocol. The presented AI-EECR protocol selects the CHs from vehicles using Quantum Chemical Reaction Optimization (QCRO) algorithm. QCRO algorithm derives a Fitness Function (FF) with the help of vehicle speed, trust level, and energy level of the vehicle. For routing process, a set of relay nodes was elected using Group Teaching Optimization Algorithm (GTOA). To validate the performance of the proposed AI-EECR protocol, a set of experiments was conducted and the results were inspected under distinct aspects.

\subsection{Paper Organization}

The upcoming sections of the paper are as follows. The clustering and routing techniques designed earlier for IoV are discussed in Section 2. Then, the proposed AI-EECR technique is elaborated in Section 3 whereas the results are validated in Section 4. At last, the conclusions are drawn in Section 5.

\section{Literature Review}

In this section, a detailed review is conducted upon state-of-the-art energy-efficient clustering and routing techniques, particularly developed for IoV.

\subsection{Prior Works on Clustering Algorithms for Io V}

Aadil et al. [14] concentrated the steadiness of IoV topology in a dynamic platform. The study presented a metaheuristic dragonfly based clustering technique named CAVDO to achieve clusterbased packet route optimization so as to create a stable topology. Further, in this study, Mobility Aware Dynamic Transmission Range algorithm (MA-DTR) was utilized for communication range adjustment based on traffic density. In order to guarantee the steadiness of self-organized communication structure, Lin et al. [15] proposed a content-aware stable multimedia communication technique for IoV. This technique controls multimedia communication within a specific range and is integrated with the communicated multimedia information of the adapted vehicle.

In Khan et al. [16], a new method based on Moth Flame Clustering Technique for IoV (MFCA-IoV) was presented. MFO is a nature simulated technique. MFCA-IoV ensures optimized clustering for reliable communication and is calculated analytically with a popular method. Grey Wolf Optimization (GWO) technique was utilized to cluster and is termed as GWOCNETs and MOPSO whereas clustering techniques that depend on ACO for VANET are CACONET and 
CLPSO. Ebadinezhad et al. [17] presented a new smart system-based method i.e., CACOIOV which stabilizes the topology with the help of metaheuristic clustering technique, depending upon the improvement of ACO in two different phases for packet route optimization. Cheng and Huang [18] developed a center-based clustering technique to support a self-organized VANET for ensuring stability of the cluster and reduce frequent changes in the state of vehicles on highways upon two metrics. A new $\mathrm{CH}$ election method was presented earlier to reduce the effect of vehicle movement variances. Gasmi et al. [19] proposed a novel Geographical Information based Clustering Algorithm 'GICA' predetermined on IoV platform.

\subsection{Prior Works on Routing Algorithms for Io V}

Zaheer et al. [20] presented a VANET architecture for smart cities which allows route selection based on real world information, established from adjacent vehicle, in ad-hoc method. The study utilized VANET architecture to implement a smart transportation system. Both preprocessing and data collection were done for distinct routes between two metropolitan cities of an emerging country. Omar et al. [21] emphasized on the improvement of a combined routing protocol for IoV setting. Reinforcement Learning (RL) and Greedy Perimeter Stateless Routing (GPSR) were combined to define a route that depends upon the need. Later, mobility method was implemented to reduce road collisions. Finally, the study concentrated on traffic management to handle mobility, loss, and delay of network and to encounter the application needs. Abbas et al. [22] proposed an optimum and cost-effective routing protocol for IoV that can overcome the challenges faced since the existing frameworks are ineffective in handling scalability and flexibility issues. As a result, the presented framework, combined the developing network standard called Software Defined Networking (SDN) in IoV. When the presented protocol was applied, it assessed the cellular network to transmit control messages from the controller at less delay.

In Yasser et al. [23], V2V execution was performed as an independent factor in ITS framework among emerging countries without Road Site Units (RSU) framework so as to overcome the present problems. Thus, the overall simulations were made for distinct VANET routing protocol with the help of Opnet simulator so as to select the optimum protocol for V2V execution. Later, an optimum V2V routing protocol was proposed depending on Key Performance Indicator while its perspectives were utilized to correlate two distinct frameworks i.e., one with V2V + RSU execution and another with the presented V2V execution. Zhang et al. [24] investigated VANET problem in sparse network settings and looked for alternatives that involve epidemic routing. The researchers proposed a BBR protocol for partial-related VANET. BBR protocol was able to endure network partitioning, owing to its low node density and high node mobility. The efficiency of BBR and epidemic routing was estimated through Geographic and Traffic Information (GTI) based mobility method which considered usual high-way situations. Furthermore, it is advantageous to have a combination of Cloud Computing (CC) technique with vehicle. This innovative technique allows the resources, applications, and data to be stored in remote servers and areas under cloud and so it is easily accessed by users with low capacity.

\section{Materials and Methods}

\subsection{System Model}

IoV possesses the characteristics of high mobility and non-uniform spatial distribution of vehicles which result in adequate topology modifications and network links' disconnectivity. To resolve these issues, a clustered IoV network is designed to offer energy-efficient and reliable communication. Fig. 1 shows the fundamental model of IoV. In generic vehicular service scenario, 
a group of vehicles is combined to form moving clusters. The clusters hold a leader called Cluster Head $(\mathrm{CH})$ which is able to manage the data regarding $\mathrm{CMs}$ and data transmission. In this research article, the following assumptions are made. Every vehicle has an identifier and an OBU. GPS service is offered to collect basic information like present position of the vehicle, velocity, and moving direction. The vehicles communicate the data among themselves via beacon signals. The beacon message gets advertised and gathered at each beacon interval that comprises of vehicle id, position, velocity, moving direction, etc.

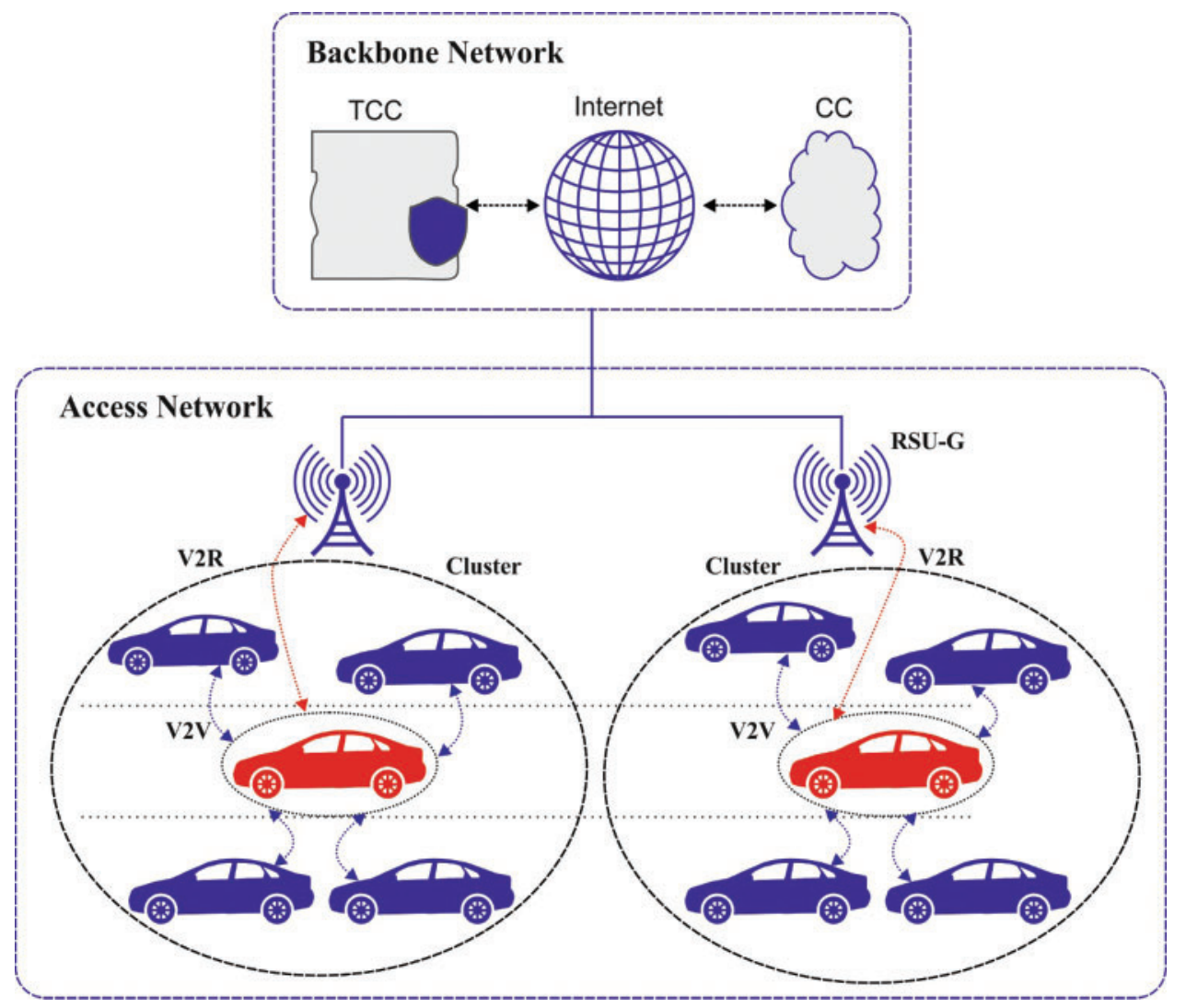

Figure 1: Network model for IoV 3.2 system architecture

Fig. 2 shows the overall architecture of the proposed model. The vehicles in the network are initialized primarily and the data regarding neighboring vehicles that exist in smart transportation environment are collected. Then, QCRO algorithm is applied to select an appropriate set of $\mathrm{CHs}$ and the clusters of vehicles are constructed. Afterwards, GTOA-based routing technique is executed to determine the relay nodes at intersection so as to determine the condition of network by allocating weights to each road segment. This way, the routes with minimal weights can be designated as the optimal path for data forwarding. The inclusion of GTOA helps in attaining optimal paths for smart transportation facilities in urban areas. Finally, the vehicles transmit the data among themselves in an energy efficient manner. 


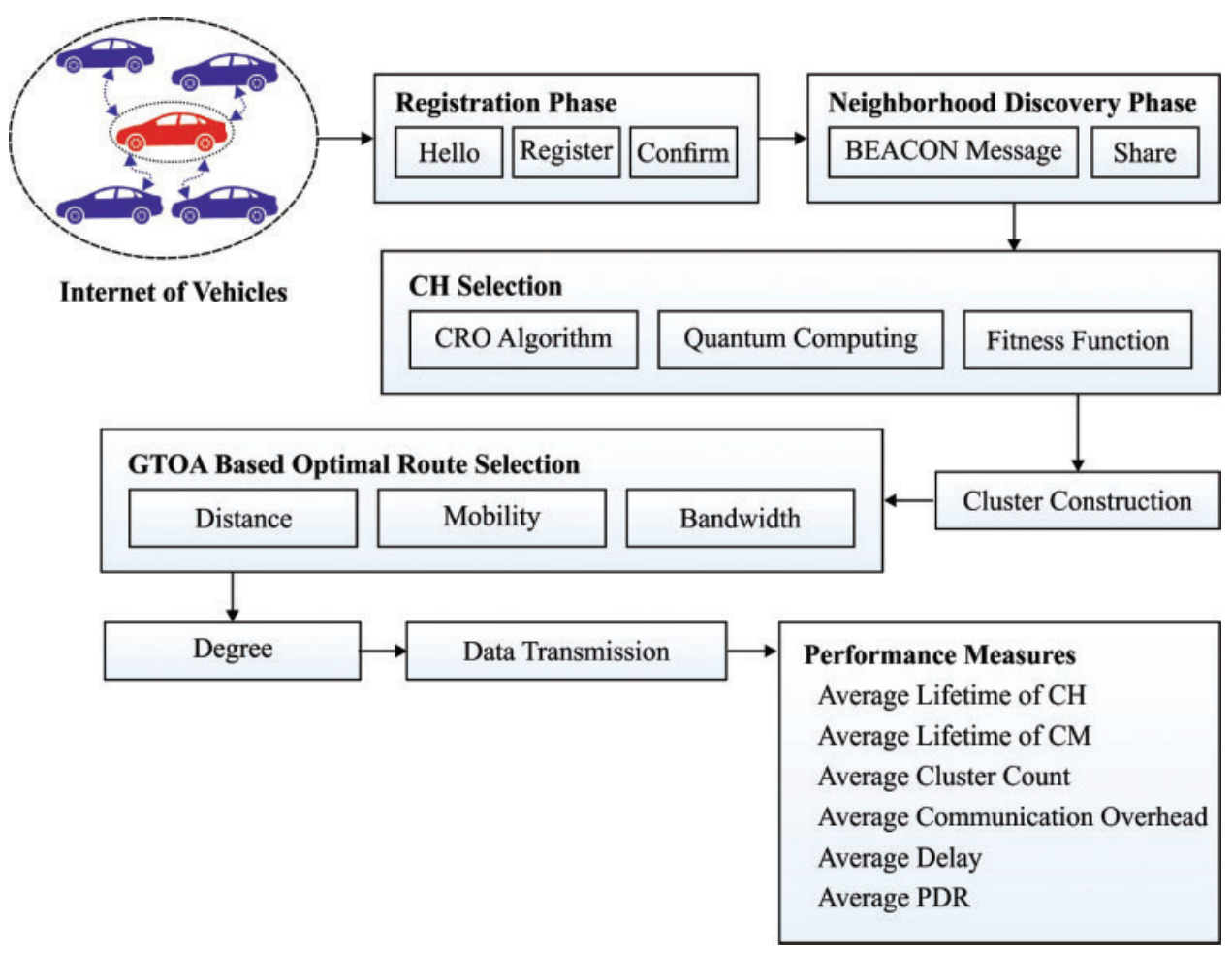

Figure 2: The overall process of the proposed method

\subsection{CH Selection Algorithm}

CRO includes a pair of collisions to ensure the occurrence of inter-molecular and unimolecular chemical reactions inside the closed container with $N_{m}$ molecule. An inter-molecular collision can create a novel molecule with a group of more than two molecules or it can ensure there is no creation of some novel molecules as a result of ineffectual inter-molecular collision. Likewise, uni-molecular collision can generate novel molecules through wall behind the decomposition of molecule or it may go ineffectually, for instance, on-wall ineffectual collision.

1) On-wall ineffectual collision: The reason is attributed to lesser perturbation from the molecules, if it hits the container wall. Assume $\varphi$ and $\varphi^{\prime}$ are the form of molecules under pre- and post-collision correspondingly. Therefore, an update is performed, when Eq. (1) is fulfilled.

$$
P E_{\phi}+K E_{\phi} \geq P E_{\phi^{\prime}}
$$

where $P E$ and $K E$ represent potential energy and kinetic energy correspondingly.

2) Decomposition: During this stage, the molecule divides into more than two molecules if the container wall is hit. Assume $\phi$ is the original form whereas the attained forms are $\phi 1^{\prime}$ and $\phi 2^{\prime}$. When original molecule and the outcome molecules fulfill Eq. (2), then the decomposition is permitted.

$$
P E_{\phi}+K E_{\phi} \geq P E_{\phi_{1}^{\prime}}+P E_{\phi_{2}^{\prime}}
$$


Generally, Eq. (2) does not succeed frequently since few parts of energy in buffer solution catalyzes the reaction as expressed in Eq. (3).

$P E_{\phi}+K E_{\phi}+$ buffer $\geq P E_{\phi_{1}^{\prime}}+P E_{\phi_{2}^{\prime}}$

When Eqs. (2) and (3) are not fulfilled, the decomposition reaction does not happen.

3) Inter-molecular ineffectual collision: During this model, more than two molecules collide with each other, producing lesser perturbations with energy transfer. Let $\phi_{1}^{\prime}$ and $\phi_{2}^{\prime}$ denote the outcome forms that are neighborhood of original forms, $\phi_{1}$ and $\phi_{2}$. Eq. (4) gets fulfilled after the implementation of modifications.

$$
P E_{\phi_{1}}+K E_{\phi_{1}}+P E_{\phi_{2}}+P E_{\phi_{2}} \geq P E_{\phi_{1}^{\prime}}+P E_{\phi_{2}^{\prime}}
$$

When Eq. (4) is not fulfilled, the molecules continue as $\phi_{1}$ and $\phi_{2}$.

4) Synthesis: A new molecule is produced during later collision between more than two molecules. This kind of collision is so drastic while its outcome creates various forms of original molecules [25]. Consider $\phi_{1}$ and $\phi_{2}$ are original molecules whereas the formation of outcome molecule is $\phi^{\prime}$. When the criteria in Eq. (5) is fulfilled, only then the synthesis occurs. Otherwise, the molecules maintain its original forms.

$$
P E_{\phi_{1}}+K E_{\phi_{1}}+P E_{\phi_{2}}+K E_{\phi_{2}} \geq P E_{\phi^{\prime}}
$$

During this chemical reaction, the population of molecules differs. This may occur due to multiple reasons such as improvement in the count of molecules from decomposition and uni-molecular collision or reduction of synthesis from intermolecular collisions. To enhance the efficiency of CRO model, Quantum Computing (QC) is used.

QC is a novel computing method that implements the models connected to quantum theory like quantum entanglement, state superposition, and quantum measurement. One of the fundamental units of QC is qubit. Two simple states $|0\rangle$ and $|1\rangle$ procedure is a qubit and is represented as a linear combination of two simple states as provided herewith.

$|Q\rangle=\alpha|0\rangle+\beta|1\rangle$

$|\alpha|^{2}$ implies the probabilities of monitoring state $|0\rangle$ and $|\beta|^{2}$ refers to probabilities of monitoring state $|1\rangle$, where $|\alpha|^{2}+|\beta|^{2}=1$. Quantum is composed of $n$ qubits. Because of the nature of quantum superposition, all quanta possess $2^{n}$ feasible values. The n-qubits quantum is referred to, as given below.

$\Psi=\sum_{x=0}^{2^{n}-1} C_{x}|x\rangle, \sum_{x=0}^{2^{n}-1}\left|C_{x}\right|^{2}=1$

A quantum gate alters the state of qubits namely, NOT gate, Hadamard gate, rotation gate, etc. Fig. 3 illustrates the flowchart of CRO technique [26].

A rotation gate is determined as given below.

$\left[\begin{array}{l}\alpha^{d}(t+1) \\ \beta^{d}(t+1)\end{array}\right]=\left[\begin{array}{ll}\cos \left(\triangle \theta^{d}\right) & -\sin \left(\triangle \theta^{d}\right) \\ \sin \left(\triangle \theta^{d}\right) & \cos \left(\triangle \theta^{d}\right)\end{array}\right]\left[\begin{array}{l}\alpha^{d}(t) \\ \beta^{d}(t)\end{array}\right]$ for $d=1,2, \ldots, n$. 


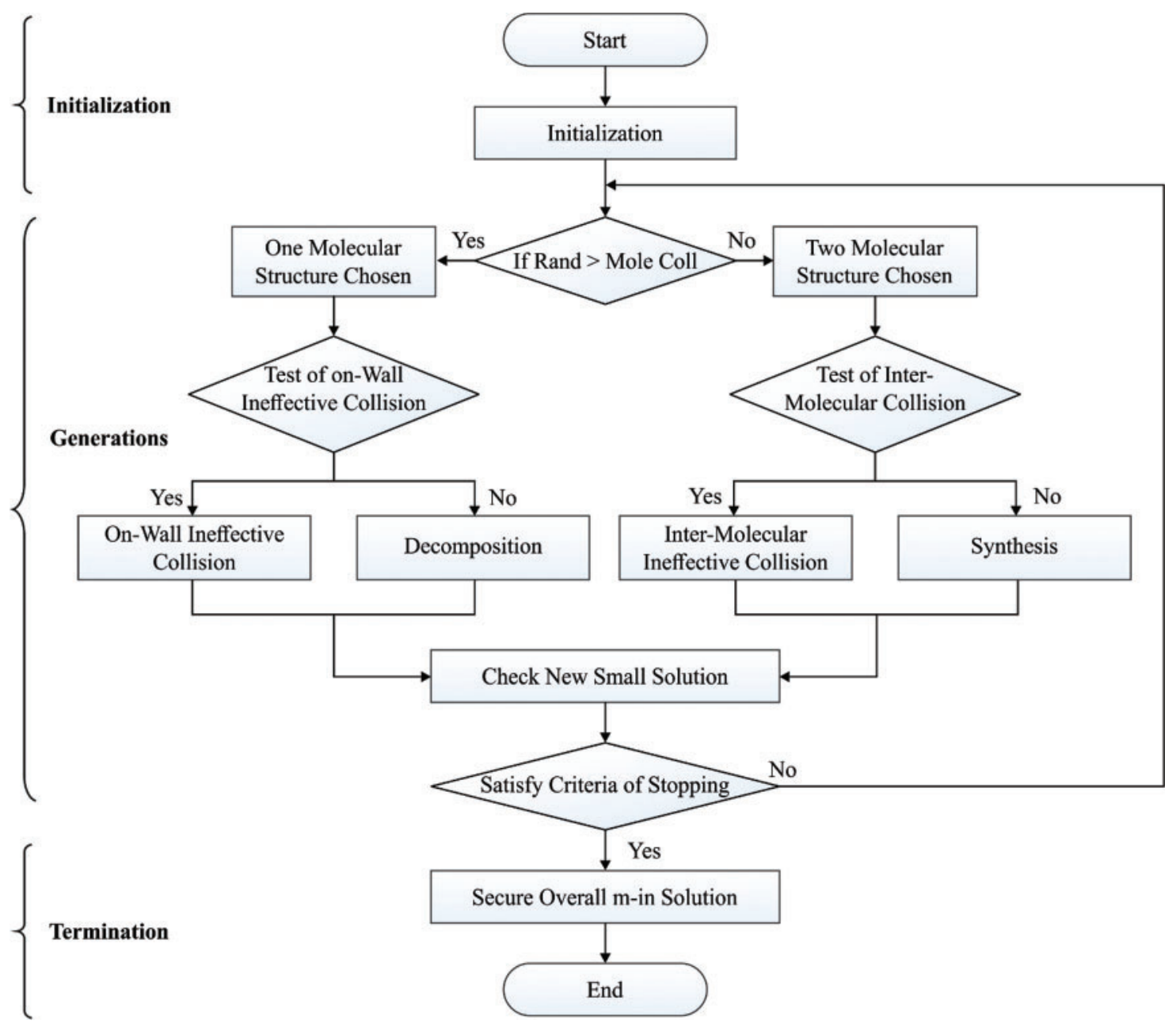

Figure 3: The flowchart of $\mathrm{CRO}$ technique

$\triangle \theta^{d}=\Delta \times S\left(\alpha^{d}, \beta^{d}\right), \Delta \theta^{d}$ implies the rotation angle of qubit, where $\Delta$ and $S\left(\alpha^{d}, \beta^{d}\right)$ denote size and way of rotation correspondingly.

The FF of QCRO algorithm, for $\mathrm{CH}$ selection in IoV, is derived in Eq. (9) and is employed as a multi-objective FF.

Fitness $_{k}=\omega 1 *$ function $_{1}+\omega 2 *$ function $_{2}$

where $\omega 1$ and $\omega 2$ denote the corresponding weights for combined FF. In QCRO, function 1 is $\Delta$ variance to clusters from route length $\alpha$. But, function 2 is obtained as a sum of CMs distance in CH's to every cluster.

function $_{1}\left(\Delta_{-} D\right)=\sum_{x=1}^{|\alpha|} \operatorname{Absolute}\left(\operatorname{degree}^{\circ}-\left|C M s_{x}\right|\right)$

where 'Absolute' is a function used to obtain absolute value, and $\left|C M s_{x}\right|$ are CMs of current cluster $\mathrm{x}$ in whole route length, $|\alpha|$ degree $^{\circ}$ is the constant value that demonstrates the degree of density to all clusters. It is identified that the user has minimum value to lower density and 
superior to denser cluster [27]. For instance, if $\Delta_{-} D$ is zero, then the clustering remains optimal. The less variance to value, results in better clustering, with respect to user specification. Function 2 FF is computed by Eq. (3) and is expressed below.

function $_{2}$ (sum of Distance)

$=\sum_{x=1}^{|\alpha|}\left(\sum_{Q}^{c H D(q)}\right.$ Eucledian Distance $\left.\left(c H D_{(q)}, c M R_{Q, q}\right)\right)$

where initial Euclidian distance is computed for every $|\alpha|$ cluster. It defines the sum of InterVehicular Nodes (IVN) $C M s_{Q, q}$ distance from $\left.c H D_{(q)}\right)$ to all clusters $(q)$ in entire clusters $|\alpha|$.

\subsection{Optimal Route Selection Algorithm}

Next to $\mathrm{CH}$ selection, GTOA is used to select the optimal route for inter-vehicle communication in IoV. The presented GTOA is simulated with group teaching method. The presented GTOA model is intended to enhance the skills of entire class by inspiring group teaching model. Assuming that several variances exist amongst students, it can be difficult to teach the group as exists in practice. There are two stages involved in the presented group teaching mechanism such as ability grouping, teacher allocation, teacher, and student stage [28]. All the four stages are explained in detail with regards to four determined principles.

\subsubsection{Ability Grouping Stage}

With no loss of generality, an entire class' skill is considered as the normal distribution, which is expressed herewith.

$f(x)=\frac{1}{\sqrt{2 \pi \delta}} e^{\frac{-(x-u)^{2}}{2 \delta^{2}}}$

where $x$ implies the essential value of the normal distribution function, $u$ represents the mean knowledge of entire class and $\delta$ refers to SD. Here, SD $\delta$ reveals the variances of skill amongst students. An optimal teacher assumes not only to enhance the mean knowledge $u$, but also decreases the SD $\delta$.

\subsubsection{Teacher Stage}

Teacher stage denotes that one student acquired the skill from his/her teacher which is equivalent to the determined second rule. A teacher creates various teaching plans for average and outstanding groups under the presented GTOA.

Teacher Stage I: The teacher concentrates on enhancing the outstanding group, owing to their strong learning skills, based on the presented GTOA and as completed in TLBO. Additionally, the teacher also attempts his/her optimal skills to improve the mean knowledge of entire class. Furthermore, the variances of accepting knowledge amongst students needs to be examined.

$\mathrm{x}_{\text {teacher }, i}^{t+1}=\mathrm{x}_{i}^{t}+a \times\left(\mathrm{T}^{t}-F \times\left(b \times \mathrm{M}^{t}+c \times \mathrm{x}_{i}^{t}\right)\right)$

$\mathrm{M}^{t}=\frac{1}{N} \sum_{i=1}^{N} \mathrm{x}_{i}^{t}$ 
$b+c=1$

where $t$ refers to the presented amount of iterations, $N$ signifies the count of students, $\mathrm{x}_{i}^{t}$ denotes the knowledge of student $i$ at time $t, \mathrm{~T}^{t}$ implies the knowledge of teacher at time $t, M^{t}$ represents the mean knowledge of the group at time $t, F$ signifies the teaching aspect which elects the teaching outcomes of teacher, $\mathrm{x}_{\text {teacher, } i}^{t+1}$ defines the knowledge of student $i$ at time $t$ learned from his/her teacher and finally $a, b$, and $c$ are three arbitrary numbers in the range of 0 to 1 .

Teacher Stage II: Assuming the worst capability of acquiring knowledge, the teacher pays heavy focus on average group to outstanding group depending on $2^{\text {nd }}$ rule, it inclines to enhance the skill of students in the viewpoint of separate student.

$\mathrm{x}_{\text {teacher }, i}^{t+1}=\mathrm{x}_{i}^{t}+2 \times d \times\left(\mathrm{T}^{t}-\mathrm{x}_{i}^{t}\right)$

where $d$ represents the arbitrary number in the range of $[0,1]$. Also, one student could not gain knowledge from teacher stage and is considered as follows (take the minimal issue as an instance).

$\mathrm{x}_{\text {teacher }, i}^{t+1}=\left\{\begin{array}{l}\mathrm{x}_{\text {teacher }, i}^{t+1}, f\left(\mathrm{x}_{\text {teacher }, i}^{t+1}\right)<f\left(\mathrm{x}_{i}^{t}\right) \\ \mathrm{x}_{i}^{t}, f\left(\mathrm{x}_{\text {teacher }, i}^{t+1}\right) \geq f\left(\mathrm{x}_{i}^{t}\right)\end{array}\right.$

\subsubsection{Student Stage}

In student stage, there involves student stage-I and student stage-II which equivalent to 3rd rule declared earlier. During spare time, one student acquires his/her skill through two models i.e., one through self-learning and other through communication with another students which are given herewith.

$\mathrm{x}_{\text {student }, i}^{t+1}=\left\{\begin{array}{l}\mathrm{x}_{\text {teacher }, i}^{t+1}+e \times\left(\mathrm{x}_{\text {teacher }, 1}^{t+1}-\mathrm{x}_{\text {teacher }, j}^{t+1}\right)+g \times\left(\mathrm{x}_{\text {teacher }, i}^{t+1}-\mathrm{x}_{i}^{t}\right), f\left(\mathrm{x}_{\text {teacher }, i}^{t+1}\right)<f\left(\mathrm{x}_{\text {teacher }, j}^{t+1}\right) \\ \mathrm{x}_{\text {teacher }, i}^{t+1}-e \times\left(\mathrm{x}_{\text {teacher }, i}^{t+1}-\mathrm{x}_{\text {teacher } . j}^{t+1}\right)+g \times\left(\mathrm{x}_{\text {teacher }, i}^{t+1}-\mathrm{x}_{i}^{t}\right), f\left(\mathrm{x}_{\text {teacher }, i}^{t+1}\right) \geq f\left(\mathrm{x}_{\text {teacher }: j}^{t+1}\right)\end{array}\right.$

where $e$ and $g$ are two arbitrary numbers in the range [0,1], $\mathrm{x}_{\text {student, } i}^{t+1}$ implies the skill of student $\mathrm{i}$ at time $\mathrm{t}$ by learning in student stage and $\mathrm{x}_{\text {student,j }}^{t+1}$ represents the skill of student $\mathrm{j}$ at time $\mathrm{t}$ acquired through learned from a teacher. Assuming that the student $j(j \in\{1,2, \ldots, i-1, i+1, \ldots, N\})$ is arbitrarily elected, in Eq. (18), $2^{\text {nd }}$ and $3^{\text {rd }}$ items on the right side denotes that the skill is acquired based on other students and self-learning correspondingly.

Also, one student could not obtain skill in student stage and is written as (take the minimal issued as in instance) follows.

$\mathrm{x}_{i}^{t+1}=\left\{\begin{array}{l}\mathrm{x}_{\text {teacher }, i}^{t+1}, f\left(\mathrm{x}_{\text {teacher }, i}^{t+1}\right)<f\left(\mathrm{x}_{\text {student }, i}^{t+1}\right) \\ \left(\mathrm{x}_{\text {student }, i}^{t+1}\right), f\left(\mathrm{x}_{\text {teacher }, i}^{t+1}\right) \geq f\left(\mathrm{x}_{\text {student }, i}^{t+1}\right)\end{array}\right.$

where $\mathrm{x}_{i}^{t+1}$ defines the knowledge of student $i$ at time $t+1$ after the learned cycle. 


\subsubsection{Teacher Allocation Stage}

According to the well-defined fourth rule, creating an optimal teacher-shared model is the most essential step to improve the skills of students. The teacher shared in the presented model is determined as given herewith.

$\mathrm{T}^{t}= \begin{cases}x_{\text {first }}^{t}, & f\left(x_{\text {first }}^{t}\right) \leq f\left(\frac{x_{\text {first }}^{t}+x_{\text {second }}^{t}+x_{\text {first }}^{t}}{3}\right) \\ \frac{x_{\text {first }}^{t}+x_{\text {second }}^{t}+x_{\text {first }}^{t}}{3}, & f\left(x_{\text {first }}^{t}\right)>f\left(\frac{x_{\text {first }}^{t}+x_{\text {second }}^{t}+x_{\text {first }}^{t}}{3}\right)\end{cases}$

where $\mathrm{x}_{\text {first }}^{t} \mathrm{x}_{\text {second }}^{t}$ and $\mathrm{x}_{\text {third }}^{t}$ are the first, second, and third optimal students correspondingly. To accelerate the convergence of the presented GTOA, average and outstanding groups shared a similar teacher.

\subsubsection{Fitness Function for Optimal Route Selection}

GTOA derives an FF to achieve data forwarding set selection. The aim of FF is to normalize the integrated effect on distance to target, node mobility, and bandwidth to select relay vehicles for data transmission. Besides, FF involves a total of four elements with individual criterion as given in Eq. (21).

$F=\operatorname{optimize}\left(\emptyset_{1} F_{1}+\emptyset_{2} F_{2}+\emptyset_{3} F_{3}+\left(1-\emptyset_{1}-\emptyset_{2}-\emptyset_{3}\right) F_{4}\right)$

where, $\emptyset_{1}, \emptyset_{2}$, and $\emptyset_{3}$ denote the weighing factors. Here, the primary element $F_{1}$ states the Euclidean distance between chemical reaction and the receiving vehicle. It is appropriate that a chemical reaction, encompassing the minimum distance toward receiving end, is chosen for subsequent forwarding vehicle. This is attained based on the fact that a transmitting vehicle can attain geographical location of the target vehicle $\left(x_{D}, y_{D}\right)$ by enquiring its location as well as based on the respective location of its neighboring vehicles in Cooperative Awareness Messages (CAM). By leveraging the location $\left(x_{n}, y_{n}\right)$ of a chemical reaction $\mathrm{n}, F_{1}$ can be defined as follows

$F_{1}=\left\|x_{n}-x_{d}\right\|=\sqrt{\left(x_{n}-x_{d}\right)^{2}+\left(y_{n}-y_{d}\right)^{2}}$

The second term $F_{2}$ portrays the mobility level of chemical reaction. To eliminate the network segmentation issue, a chemical reaction with least mobility is referred. The average speed for a chemical reaction in recent time $T_{S}$ can be defined as follows

$F_{2}=\frac{1}{T_{s}} \sum_{t_{s}=1}^{T_{s}} \sqrt{\left(x_{t_{s}}-x_{t_{s-1}}\right)^{2}+\left(y_{t_{s}}-y_{t_{s-1}}\right)^{2}}$

where $\left(x_{t_{s}}, y_{t_{s}}\right)$ and $\left(x_{t_{s-1}}, y_{t_{s-1}}\right)$ denote the geographical positions of chemical reaction at time $t_{s}$ and $t_{s-1}$ respectively.

In order to improve QoS, bandwidth is an important parameter that is treated as $3^{\text {rd }}$ term $F_{3}$ during optimization. To determine the bandwidth level, the Available Bandwidth Estimator (ABE) scheme is employed. ABE generalizes channel monitoring to compute the occupancy ratio of nodes with detached emission, probabilistic incorporation of values to synchronize nodes, approximation of collision likelihood between the nodes, and distinct overhead.

$F_{3}=(1-A) \cdot\left(1-p_{c}\right) \cdot t_{s} \cdot t_{r} \cdot B$ 
where $A$ means excessive overhead owing to binary exponential backoff model and $p_{c}$ infers the collision likelihood of hello packets. $t_{s}$ and $t_{r}$ indicate the idle timing of transmitting and receiving ends correspondingly. $B$ indicates the ability of link between transmitter and the receiver. The concluding element $F_{4}$ offers a degree associated with chemical reaction. At that time, $F_{4}$ can be defined as follows.

$F_{4}=\left|N_{d e g}\right|$

where, $\left|N_{d e g}\right|$ specifies the number of neighboring vehicles. The vehicle located near the optimum location is chosen as the forwarding vehicle for communication.

\section{Performance Validation}

\subsection{Implementation Setup}

The presented technique was simulated in an environment combining NS-2 simulation tool and VanetMobiSim. The mobility was experimented on one-directional roadway spanning $6 \mathrm{~km}$ length with three lanes and two RSU-G. The parameters, utilized in the simulation, are listed in Tab. 1. An area of $6000 \mathrm{~m} \times 50 \mathrm{~m}$ was utilized to simulate 60 to 80 vehicles. The vehicles travel with a velocity of 10 to $35 \mathrm{~m} / \mathrm{s}$ while the highest velocity was maintained at $40 \mathrm{~m} / \mathrm{s}$. In order to validate the efficiency of the proposed EHCP, the parameters were tested for N-hop, and DMCNF too.

Table 1: Simulation settings

\begin{tabular}{ll}
\hline Parameter & Value \\
\hline Simulation time & $360 \mathrm{~s}$ \\
Area & $6000 \mathrm{~m} \times 50 \mathrm{~m}$ \\
Communication range & $100-300 \mathrm{~m}$ \\
No. of RSU-G & 2 \\
Vehicle density & $60-180$ \\
Velocity & $10-35 \mathrm{~m} / \mathrm{s}$ \\
\hline
\end{tabular}

\subsection{Results Analysis}

Tab. 2 shows the results obtained from a detailed comparison of the presented AI-EECR technique against existing models [9] under varying velocity rates. Fig. 4 demonstrates the average lifetime of $\mathrm{CH}(\mathrm{ALCH})$ analysis of AI-EECR model under distinct velocities. The figure demonstrates that AI-EECR model gained the maximum ALCH over other methods under different velocity rates.

For instance, under the velocity of $10 \mathrm{~m} / \mathrm{s}$, AI-EECR model attained a high ALCH of 185 , whereas other methods such as N-hop, DMCNF, and HCP-MIV models obtained the least ALCH values such as 94, 142, and 172 respectively. Likewise, under the velocity of $20 \mathrm{~m} / \mathrm{s}$, the presented AI-EECR method achieved a maximum ALCH of 187, whereas N-hop, DMCNF, and HCP-MIV methodologies accomplished minimum ALCH values such as 90, 130, and 163 respectively. Similarly, under the velocity of $30 \mathrm{~m} / \mathrm{s}$, AI-EECR technique attained a high ALCH of 175, whereas N-hop, DMCNF, and HCP-MIV models attained least ALCH values such as 85, 123, and 155 correspondingly. Fig. 5 showcases the results for Average Lifetime of CM (ALCM) 
analysis obtained by AI-EECR method under distinct velocity rates. The figure exhibits that AIEECR method gained the maximum ALCM over other methods under different velocity rates. For instance, under the velocity of $10 \mathrm{~m} / \mathrm{s}$, AI-EECR approach attained a superior ALCM of 225, whereas the N-hop, DMCNF, and HCP-MIV models obtained the least ALCM values such as 132, 175, and 199 respectively. In addition, under the velocity of $20 \mathrm{~m} / \mathrm{s}$, AI-EECR model achieved a high ALCM of 229, whereas N-hop, DMCNF, and HCP-MIV models obtained the least ALCM values such as 109, 178, and 199 correspondingly. Also, under the velocity of $30 \mathrm{~m} / \mathrm{s}$, AI-EECR model attained a high ALCM of 239, whereas N-hop, DMCNF, and HCP-MIV models obtained the least ALCM values such as 89, 182, and 190 correspondingly.

Table 2: Result of the analysis of proposed and the existing methods under distinct measures

\begin{tabular}{lllll}
\hline \multicolumn{4}{l}{ Average lifetime of cluster head vs. velocity } \\
\hline Velocity (m/s) & N-hop & DMCNF & HCP-MIV & AI-EECR \\
\hline 10 & 94 & 142 & 172 & 185 \\
15 & 91 & 129 & 165 & 192 \\
20 & 90 & 130 & 163 & 187 \\
25 & 88 & 127 & 160 & 190 \\
30 & 85 & 123 & 155 & 175 \\
35 & 75 & 115 & 153 & 184
\end{tabular}

Average lifetime of cluster member vs. velocity

\begin{tabular}{lllll}
\hline Velocity (m/s) & N-hop & DMCNF & HCP-MIV & AI-EECR \\
\hline 10 & 132 & 175 & 199 & 225 \\
15 & 130 & 177 & 200 & 236 \\
20 & 109 & 178 & 199 & 229 \\
25 & 93 & 180 & 198 & 234 \\
30 & 89 & 182 & 190 & 239 \\
35 & 75 & 180 & 188 & 228 \\
\hline
\end{tabular}

Average cluster count vs. velocity

\begin{tabular}{lllll}
\hline Velocity $(\mathrm{m} / \mathrm{s})$ & N-hop & DMCNF & HCP-MIV & AI-EECR \\
\hline 10 & 8.60 & 8.50 & 6.30 & 5.20 \\
15 & 8.70 & 8.60 & 6.50 & 5.30 \\
20 & 8.80 & 8.70 & 6.60 & 5.50 \\
25 & 8.90 & 8.80 & 6.80 & 5.50 \\
30 & 9.20 & 9.00 & 6.90 & 5.70 \\
35 & 10.00 & 8.90 & 7.00 & 6.30 \\
\hline
\end{tabular}

Fig. 6 portrays the outcomes of average cluster count analysis obtained by AI-EECR model in terms of average cluster count. From the figure, it is obvious that the proposed AI-EECR model performed well and obtained minimal cluster count under all velocity rates. For instance, on the applied velocity of $10 \mathrm{~m} / \mathrm{s}$, AI-EECR model offered the least average cluster count of 5.2, 
whereas other methods namely, N-hop, DMCNF, and HCP-MIV models achieved high average cluster counts such as $8.6,8.5$, and 6.3 respectively.

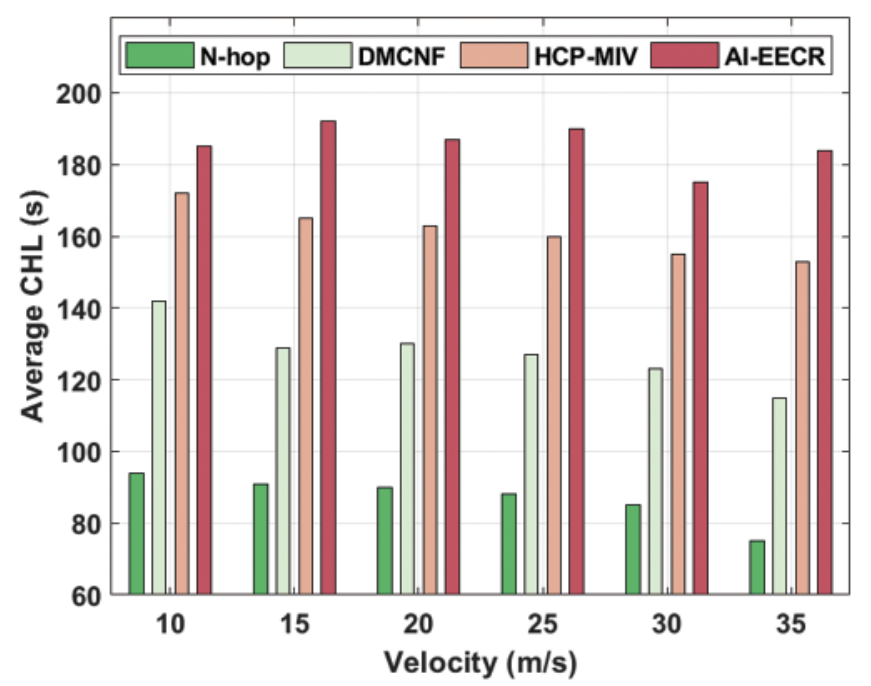

Figure 4: Average CHL analysis of AI-EECR model

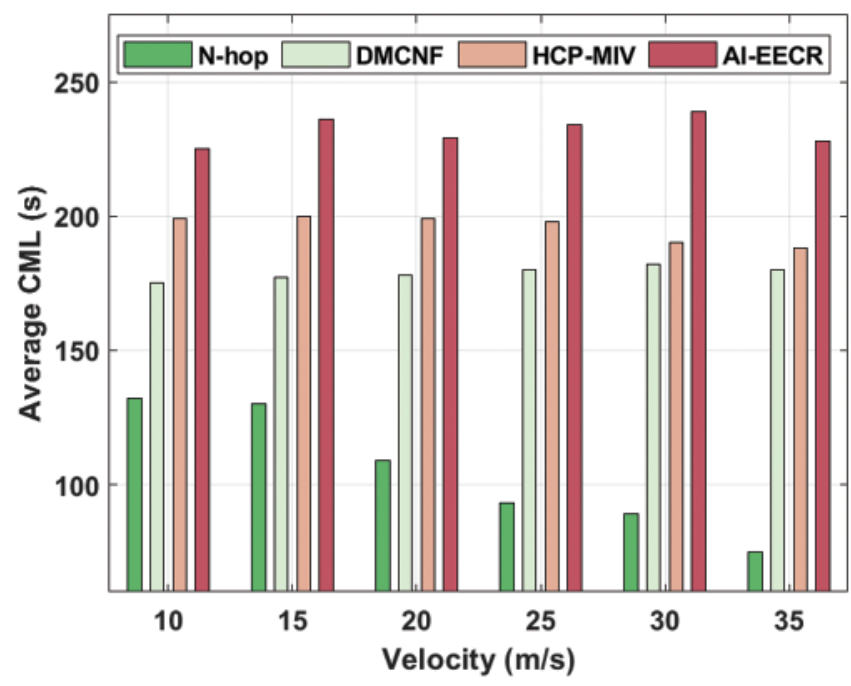

Figure 5: Average CML analysis of AI-EECR model

Fig. 7 shows the results for average Communication Overhead (CO) analysis of the proposed AI-EECR method under distinct velocity rates. The figure demonstrates that AI-EECR approach gained a low average $\mathrm{CO}$ over other methods under different velocity rates. For instance, under the velocity of $10 \mathrm{~m} / \mathrm{s}$, AI-EECR method attained a minimum average $\mathrm{CO}$ of 8.51 , whereas $\mathrm{N}$ hop, DMCNF, and HCP-MIV models attained high average $\mathrm{CO}$ values such as 17, 14, and 9.56 correspondingly. Besides, under the velocity of $20 \mathrm{~m} / \mathrm{s}$, AI-EECR model attained a low average $\mathrm{CO}$ of 9.32, whereas N-hop, DMCNF, and HCP-MIV models obtained high average CO values 
being 19, 14.60, and 11 respectively. Furthermore, under the velocity of $30 \mathrm{~m} / \mathrm{s}$, AI-EECR model attained a less average CO of 10.14, whereas N-hop, DMCNF, and HCP-MIV models obtained superior average $\mathrm{CO}$ levels such as 22,15 , and 12.5 respectively.

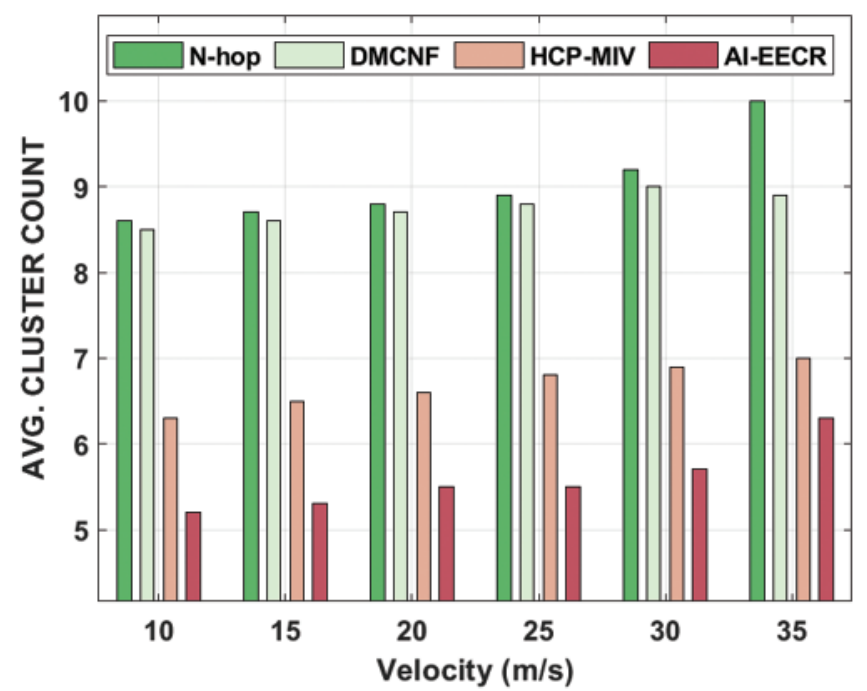

Figure 6: Average cluster count analysis of AI-EECR model

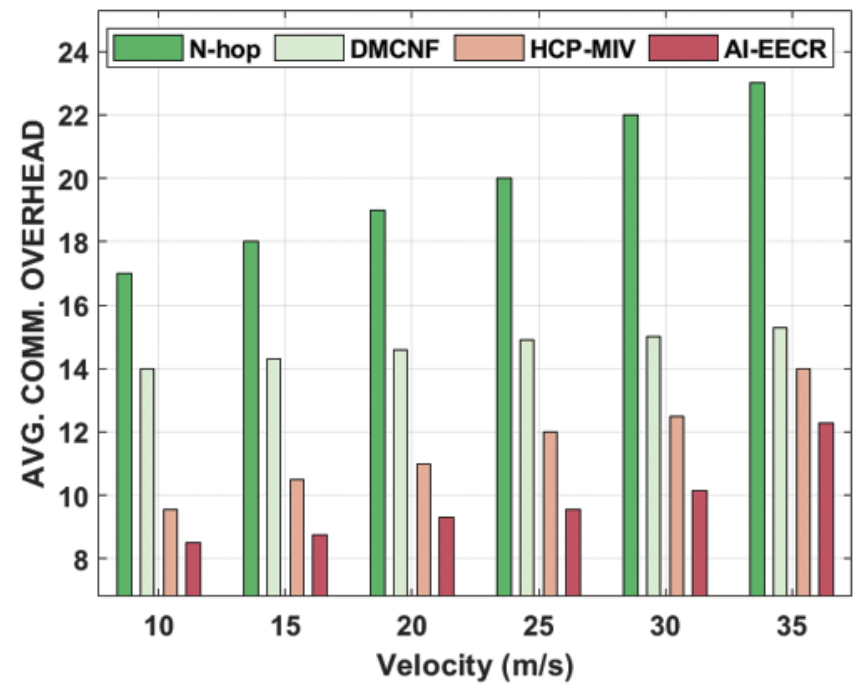

Figure 7: Average communication overhead analysis of AI-EECR model

8 demonstrates the results for average delay analysis obtained by AI-EECR technique under distinct vehicles. The figure portrays that AI-EECR model gained a minimum average delay over other models, under count of vehicles. For instance, under 60 vehicles, AI-EECR technique attained a minimal average delay of 1750, whereas N-hop, DMCNF, and HCP-MIV methodologies attained high average delays such as 4600, 3500, and 2550 respectively. In the meantime, under 120 vehicles, AI-EECR model attained a low average delay of 1080, whereas N-hop, DMCNF, 
and HCP-MIV models obtained high average delays such as 3200, 1600, and 1350 respectively. Simultaneously, under 180 vehicles, AI-EECR algorithm attained a less average delay of 430, whereas N-hop, DMCNF, and HCP-MIV models reached superior average delays such as 2200, 1100, and 550 correspondingly as shown in Fig. 8.

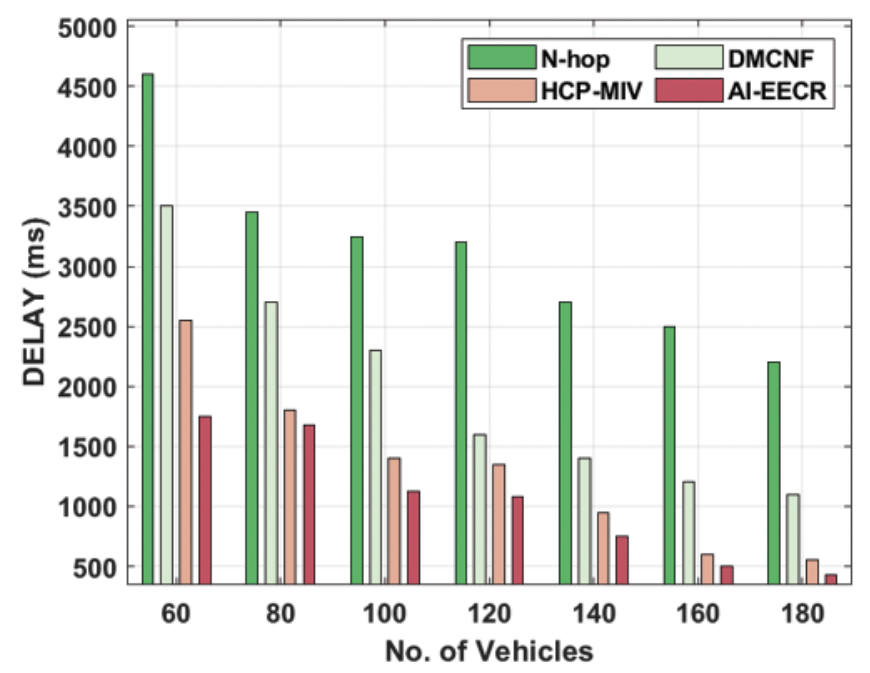

Figure 8: Average delay analysis of AI-EECR model

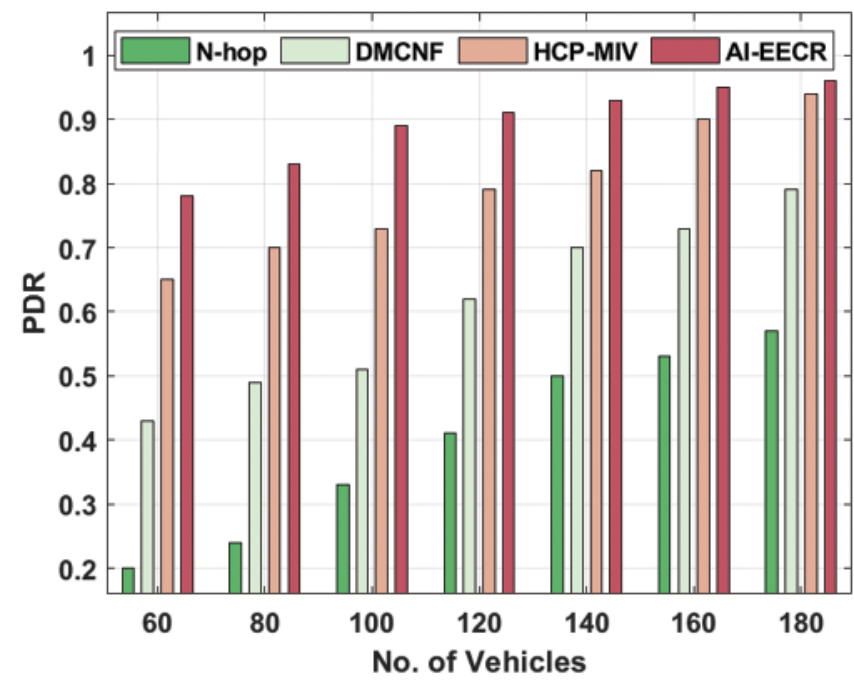

Figure 9: Average PDR analysis of AI-EECR model

Fig. 9 exhibits the results of Average PDR (APDR) analysis obtained by AI-EECR algorithm under different vehicles. The figure demonstrates that AI-EECR model gained the maximum APDR over other methods under different count of vehicles. For instance, under 60 vehicles, AI-EECR model achieved a high APDR of 0.78, whereas N-hop, DMCNF, and HCP-MIV techniques obtained low APDR values such as $0.20,0.43$, and 0.65 correspondingly. Additionally, under 120 vehicles, AI-EECR model attained a high APDR of 0.91, whereas N-hop, DMCNF, 
and HCP-MIV models achieved low APDR values being 0.41, 0.62, and 0.79 respectively. At the same time, under 180 vehicles, AI-EECR model attained a high APDR of 0.96, whereas N-hop, DMCNF, and HCP-MIV algorithms obtained the least APDR values of 0.57, 0.79, and 0.94 correspondingly. The enhanced process is due to the integration of QCRO based $\mathrm{CH}$ selection, and GTOA based routing protocol.

\section{Conclusion}

The current research article developed an energy-efficient and reliable cluster-based routing protocol named AI-EECR for IoV in urban computing. The proposed AI-EECR protocol operates under three stages namely, network initialization, $\mathrm{CH}$ selection, and routing protocol. Once the vehicles are initialized, $\mathrm{CH}$ selection process occurs effectively by QCRO algorithm. Besides, GTOA-based routing technique is implemented for optimal selection of routes for intervehicular communication. The inclusion of GTOA helps in attaining the optimal path for smart transportation facilities in urban areas. In order to validate the performance of the proposed AIEECR protocol, a set of experiments was carried out and the results were inspected under distinct aspects. The experimental outcomes established that AI-EECR model outperformed the existing methods under different measures. Particularly, with the existence of 180 vehicles, AI-EECR model attained a high APDR of 0.96. The future works are suggested to concentrate on the adoption of trust-based framework in IoV networks. Besides, privacy preservation techniques can also be designed in order to ensure security in IoV.

Funding Statement: The authors extend their appreciation to the Deanship of Scientific Research at King Khalid University for funding this work under grant number (RGP 2/25/42), Received by Fahd N. Al-Wesabi. www.kku.edu.sa. This research was funded by the Deanship of Scientific Research at Princess Nourah bint Abdulrahman University through the Fast-track Research Funding Program.

Conflicts of Interest: The authors declare that they have no conflicts of interest to report regarding the present study.

\section{References}

[1] A. Nikitas, K. Michalakopoulou, E. T. Njoya and D. Karampatzakis, "Artificial intelligence, transport and the smart city: Definitions and dimensions of a new mobility era," Sustainability, vol. 12, no. 7, pp. 2789, 2020.

[2] R. D. Knowles, F. Ferbrache and A. Nikitas, "Transport's historical, contemporary and future role in shaping urban development: Re-evaluating transit oriented development," Cities, vol. 99, pp. 102607, 2020.

[3] V. Albino, U. Berardi and R. M. Dangelico, "Smart cities: Definitions, dimensions, performance, and initiatives," Journal of Urban Technology, vol. 22, no. 1, pp. 3-21, 2015.

[4] F. Creutzig, P. Jochem, O. Y. Edelenbosch, L. Mattauch, D. P. V. Vuuren et al., "Transport: A roadblock to climate change mitigation?," Science, vol. 350, no. 6263, pp. 911-912, 2015.

[5] J. Cheng, J. Cheng, M. Zhou, F. Liu, S. Gao et al., "Routing in internet of vehicles: A review," IEEE Transactions on Intelligent Transportation Systems, vol. 16, no. 5, pp. 2339-2352, 2015.

[6] S. K. Lakshmanaprabu, K. Shankar, S. S. Rani, E. Abdulhay, N. Arunkumar et al., "An effect of big data technology with ant colony optimization based routing in vehicular ad hoc networks: Towards smart cities," Journal of Cleaner Production, vol. 217, pp. 584-593, 2019. 
[7] H. Wu, H. Tang and L. Dong, "A novel routing protocol based on mobile social networks and internet of vehicles," in Int. Conf. on Internet of Vehicles IOV 2014. Proc.: Lecture Notes in Computer Science Book Series, (LNCS), New York City, NY, USA, vol. 8662, pp. 1-10, 2014.

[8] O. Senouci, Z. Aliouat and S. Harous, "A review of routing protocols in internet of vehicles and their challenges," Sensor Review, vol. 39, no. 1, pp. 58-70, 2019.

[9] A. K. Dutta, M. Elhoseny, V. Dahiya and K. Shankar, "An efficient hierarchical clustering protocol for multihop internet of vehicles communication," Transactions on Emerging Telecommunications Technologies, vol. 31, no. 5, pp. 1-13, 2020.

[10] C. J. Huaug, Y. W. Wang, H. M. Chen, A. L. Cheng, J. J. Jian et al., "An adaptive multimedia streaming dissemination system for vehicular networks," Applied Soft Computing, vol. 13, no. 12, pp. 4508-4518, 2013.

[11] H. Fatemidokht and M. Kuchaki Rafsanjani, "QMM-Vanet: An efficient clustering algorithm based on QoS and monitoring of malicious vehicles in vehicular ad hoc networks," Journal of Systems and Software, vol. 165, pp. 110561, 2020.

[12] S. Arjunan, S. Pothula and D. Ponnurangam, "F5n-based unequal clustering protocol (F5NUCP) for wireless sensor networks," International Journal of Communication Systems, vol. 31, no. 17, pp. e3811, 2018.

[13] M. Buvanesvari, J. Uthayakumar and J. Amudhavel, "Fuzzy based clustering to maximize network lifetime in wireless mobile sensor networks," Journal of Advanced Research in Dynamical and Control Systems, vol. 9, no. 12, pp. 2156-2167, 2017.

[14] F. Aadil, W. Ahsan, Z. U. Rehman, P. A. Shah, S. Rho et al., "Clustering algorithm for internet of vehicles (IoV) based on dragonfly optimizer (CAVDO)," The Journal of Supercomputing, vol. 74, no. 9, pp. 4542-4567, 2018.

[15] K. Lin, F. Xia and G. Fortino, "Data-driven clustering for multimedia communication in internet of vehicles," Future Generation Computer Systems, vol. 94, pp. 610-619, 2019.

[16] M. F. Khan, F. Aadil, M. Maqsood, S. H. R. Bukhari, M. Hussain et al., "Moth flame clustering algorithm for internet of vehicle (MFCA-ioV)," IEEE Access, vol. 7, pp. 11613-11629, 2019.

[17] S. Ebadinezhad, Z. Dereboylu and E. Ever, "Clustering-based modified ant colony optimizer for internet of vehicles (CACOIOV)," Sustainability, vol. 11, no. 9, pp. 2624, 2019.

[18] X. Cheng and B. Huang, "A center-based secure and stable clustering algorithm for vanets on highways," Wireless Communications and Mobile Computing, vol. 2019, pp. 1-10, 2019.

[19] R. Gasmi, M. Aliouat and H. Seba, "Geographical information based clustering algorithm for internet of vehicles," in Machine Learning for Networking: Third Int. Conf., MLN 2020. Lecture Notes in Computer Science Book Series (LNCS), Paris, France, vol. 12629, pp. 107-121, 2020.

[20] T. Zaheer, A. W. Malik, A. U. Rahman, A. Zahir and M. M. Fraz, "A vehicular network-based intelligent transport system for smart cities," International Journal of Distributed Sensor Networks, vol. 15, no. 11, pp. 155014771988884, 2019.

[21] N. Omar, N. Yaakob, Z. Husin and M. Elshaikh, "Design and development of greedlea routing protocol for internet of vehicle (iov)," IOP Conference Series: Materials Science and Engineering, vol. 767, pp. 012034, 2020.

[22] M. T. Abbas, A. Muhammad and W.-C. Song, "SD-Iov: SDN enabled routing for internet of vehicles in road-aware approach," Journal of Ambient Intelligence and Humanized Computing, vol. 11, no. 3, pp. 1265-1280, 2020.

[23] A. Yasser, M. Zorkany and N. Abdel Kader, "VANET routing protocol for V2V implementation: A suitable solution for developing countries," Cogent Engineering, vol. 4, no. 1, pp. 1362802, 2017.

[24] M. Zhang and R. Wolff, "Routing protocols for vehicular Ad Hoc networks in rural areas," IEEE Communications Magazine, vol. 46, no. 11, pp. 126-131, 2008.

[25] P. C. Srinivasa Rao, A. J. Sravan Kumar, Q. Niyaz, P. Sidike and V. K. Devabhaktuni, "Binary chemical reaction optimization based feature selection techniques for machine learning classification problems," Expert Systems with Applications, vol. 167, pp. 114169, 2021. 
[26] R. K. Yadav and H. Banka, "An improved chemical reaction-based approach for multiple sequence alignment," Current Science, vol. 112, no. 3, pp. 527, 2017.

[27] W. Ahsan, M. F. Khan, F. Aadil, M. Maqsood, S. Ashraf et al., "Optimized node clustering in vanets by using meta-heuristic algorithms," Electronics, vol. 9, no. 3, pp. 394, 2020.

[28] Y. Zhang and Z. Jin, "Group teaching optimization algorithm: A novel metaheuristic method for solving global optimization problems," Expert Systems with Applications, vol. 148, pp. 113246, 2020. 